\title{
Conformal Weyl-Euler-Lagrangian equations on 4-Walker manifolds
}

\author{
Zeki Kasap \\ Department of Elementary Education, Faculty of Education, Pamukkale University, Denizli, Turkey \\ Received: 29 December 2015, Revised: 29 December 2015, Accepted: 9 January 2016 \\ Published online: 10 April 2016
}

\begin{abstract}
The main purpose of the present paper is to study almost complex structures conformal Weyl-Euler-Lagrangian equations on 4-dimensional Walker manifolds for (conservative) dynamical systems. In this study, routes of objects moving in space will be modeled mathematically on 4-dimensional Walker manifolds that these are time-dependent partial differential equations. A Walker $\mathrm{n}$-manifold is a semi-Riemannian n-manifold, which admits a field of parallel null $r$-planes, with $\mathrm{r} \leq \frac{n}{2}$. It is well-known that semi-Riemannian geometry has an important tool to describe spacetime events. Therefore, solutions of some structures about 4-Walker manifold can be used to explain spacetime singularities. Then, here we present complex analogues of Lagrangian mechanical systems on 4-Walker manifold. Also, the geometrical-physical results related to complex mechanical systems are also discussed for conformal Weyl-EulerLagrangian equations for (conservative) dynamical systems and solution of the motion equations using Maple Algebra software will be made.
\end{abstract}

Keywords: Walker Manifolds, Weyl theory, holomorphic, symplectic geometry, conformal geometry, Lagrangian, mechanical system, Riemannian manifold, almost complex manifolds.

\section{Introduction}

It is well-known that an electromagnetic field is a physical field produced by electrically charged objects on the living space. How the movement of objects in electrical, magnetically and gravitational fields force is very important. For instance, on a weather map or the surface wind velocity is describes by assigning a vector to each point on a map. Also, each vector represents the speed and direction of the movement of air or surface at that point on defined space. A classical field theory explains the study of how one or more physical fields interact with matter which is used quantum and classical mechanics of physics branches.

Differential geometry is widely used as a mathematical operative branch for classical field theory. It known using the techniques of differential calculus, integral calculus, linear algebra and multilinear algebra to study problems in geometry. Also, a dynamical system is a concept in mathematics where a fixed rule describes how a point in a geometrical space depends on time. At any given time a dynamical system has a state given by a set of real numbers (a vector) that can be represented by a point in an appropriate state space or a geometrical manifold. In addition, a dynamical systems theory is an area of mathematics used to describe the behavior of complex dynamical systems, usually by employing differential equations or difference equations. The dynamic equations used to model mechanical systems. A model is a precise representation of a system's dynamics used to answer questions via analysis and simulation. The dynamic equations for moving objects are obtained for Lagrangian mechanics by many authors in many areas [1]-[3]. Kasap and Tekkoyun found Lagrangian and Hamiltonian formalism for mechanical systems using para/pseudo-Kähler manifolds, representing an interesting multidisciplinary field of research [4]. Kasap introduced Weyl-Euler-Lagrange equations of motion on flat manifold [5]. Walker shown that a Walker n-manifold, they mean a 
semi-Riemannian manifold which admits a field of parallel null $r$-planes, with $r \leq n / 2$ [6]. Salimov et al examined that a Walker 4-manifold is a pseudo-Riemannian manifold, $\left(M_{4}, g\right)$ of neutral signature, which admits a field of parallel null 2-plane [7]. Matsushita studied that a Walker 4-manifold, endowed with a canonical neutral metric depending on three arbitrary functions, admits a specific almost complex structure and an associated opposite almost complex structure [8]. Garcia-Rio et al showed that such a Walker 4-manifold can carry various structures with respect to a certain kind of almost complex structure, e.g., symplectic structures, Kähler structures, Hermitian structures, according as the properties of certain functions which define the canonical form of the metric [9]. Batat et al studied the curvature properties of a large class of four-dimensional Walker metrics [10]. Nadjafikhah and Jafari constructed that Lie symmetry group method is applied to find the lie point symmetries group of a PDE system that is determined general form of four-dimensional Einstein Walker manifold [11]. Salimov and Iscan showed that a Walker 4-manifold is a semi-Riemannian manifold $\left(M_{4}, g\right)$ of neutral signature, which admits a field of parallel null 2-plane [12]. Brozos-Vazquez et al examined commutativity properties of the Ricci operator, of the skew-symmetric curvature operator, and of the Jacobi operator for certain Walker manifolds of signature (2,2) [13]. Davidov showed that any proper almost Hermitian structure on a Walker 4-manifold is isotropic Kähler [14]. Tekkoyun showed that a Walker n-manifold is a semi-Riemannian n-manifold, which admits a field of parallel null $r$-planes, with $r \leq n / 2$ [15]. Law examined that a four-dimensional Walker geometry is a four-dimensional manifold $M$ with a neutral metric $g$ and a parallel distribution of totally null two-planes [16]. Ghanam and Thompson submitted that special interest manifolds are Walker manifolds of even dimensions $(n=2 m)$ admitting a field of null planes of maximum dimensionality $(r=m)$ [17].

\section{Preliminaries}

Definition 1. Walker manifold is a triple $(M, g, D)$ where $M$ is an $n$-dimensional manifold, $g$ an indefinite metric and $D$ an r-dimensional parallel null distribution [15].

Of special interest are those manifolds admitting a field of null planes of maximum dimensionality $r=n / 2$. Since the dimension of a null plane is source $r \leq n / 2$, the lowest possible case is that of $(+,+,-,-)$-manifolds admitting a field of parallel null 2-planes.

Definition 2. A metric tensor is a non-degenerate, smooth, symmetric, bilinear map which assigns a real number to pairs of tangent vectors at each tangent space of the manifold. Denoting the metric tensor $g$ we can express this as $g: T_{p} M \times T_{p} M \rightarrow \mathbb{R}$. The map is symmetric and bilinear so if $X, Y, Z \in T_{p} M$ are tangent vectors at a point $p$ to the manifold $M$ then we have

(1) $g(X, Y)=g(Y, X)$,

(2) $g(a X+Y, Z)=a g(X, Z)+g(Y, Z)$ for any real number $a \in \mathbb{R}[6]$.

Definition 3. A pseudo-Riemannian manifold (also called a semi-Riemannian manifold) (M, $g$ ) is a differentiable manifold $M$ equipped with a non-degenerate, smooth, symmetric metric tensor $g$. Such a metric is called a pseudo-Riemannian metric and its values can be positive, negative or zero. The signature of a pseudo-Riemannian metric is $(p, q)$, where both $p$ and $q$ are non-negative. The model space for a pseudo-Riemannian manifold of signature ( $p, q)$ is $\mathbb{R}^{p, q}$ with the metric $g=d x_{1}^{2}+d x_{2}^{2}+\ldots+d x_{p}^{2}-d x_{p+1}^{2}-\ldots-d x_{p+q}^{2}[14]$.

Definition 4. Let $M$ be a pseudo-Riemannian manifold of signature $(p, q)$. We suppose given a splitting of the tangent bundle in the form $T M=V_{1} \oplus V_{2}$ where $V_{1}$ and $V_{2}$ are smooth subbundles which are called distributions. If $M$ is Riemannian, we can take $V_{2}=V_{1}^{\perp}$ to be the orthogonal complement of $V_{1}$ and in that case $V_{2}$ is again parallel. In the pseudo-Riemannian setting, of course, $V_{2} \cap V_{1}$ need not be trivial and there exist examples where although $V_{1}$ is parallel, there exists no complementary parallel distribution. Let $V_{1}$ be a parallel distribution. The rank of $g$ restricted to $V_{1}$ is constant. We can say that $V_{1}$ is a null parallel distribution if $V_{1}$ is parallel and if the metric restricted to $V_{1}$ vanishes identically [14]. 
Proposition 1. A neutral metric $g$ on a 4-manifold $M_{4}$ is said to be Walker metric if there exists a 2-dimensional null distribution $\mathrm{D}$ on $\mathrm{M}_{4}$, which is parallel with respect to g. From Walker theorem there is a system of coordinates with respect to which g takes the local canonical form

$$
g\left(x_{1}, x_{2}, x_{3}, x_{4}\right)=\left(\begin{array}{cccc}
0 & 0 & 1 & 0 \\
0 & 0 & 0 & 1 \\
1 & 0 & a & c \\
0 & 1 & c & b
\end{array}\right)
$$

where $a, b, c$ are smooth functions of the coordinates $\left(x_{1}, x_{2}, x_{3}, x_{4}\right)$. Also, $h_{x_{1}, \ldots, x_{r}}$ means partial derivatives $\frac{\partial h}{\partial_{x_{1}} \ldots \partial_{x_{x}}}$ for any function $h\left(x_{1}, x_{2}, x_{3}, x_{4}\right)$. The parallel null 2-plane $D$ is spanned locally by $\left\{\partial_{x_{1}}, \partial_{x_{2}}\right\}$, where $\partial_{x_{i}}$ are abbreviated forms of $\partial_{x_{1}}=\partial / \partial_{x_{1}}, . ., \partial_{x_{4}}=\partial / \partial_{x_{4}}[6,15]$. Walker [6] studied pseudo-Riemannian manifolds $M$ with a parallel field of null planes $D$ and derived a canonical form. Motivated by this seminal work, one says that a pseudo-Riemannian manifold $M$ which admits a null parallel i.e., (degenerate) distribution D is a Walker manifold.

\section{The theory of $J$-holomorphic curves}

Definition 5. J-holomorphic curve is a smooth map from a Riemann surface into an almost complex manifold that satisfies the Cauchy-Riemann equation.

Definition 6. Let $\omega$ be a 2-form on a manifold $M$. For each point $p \in M$, the map $\omega_{p}: T p M \times T p M \rightarrow R$ is skew-symmetric and bilinear on the tangent space to $M$ at $p$, and $\omega_{p}$ varies smoothly in $p$.

Definition 7. The 2-form $\omega$ is symplectic if $\omega$ is closed (its exterior derivative is $d \omega=0$ ) and $\omega_{p}$ is symplectic for all $p \in$ M. A symplectic manifold is a pair $(M, \omega)$ where $M$ is a manifold and $\omega$ is a symplectic form. Symplectic manifolds must be even-dimensional. Let $M=\mathbb{R}^{2 n}$ with linear coordinates $x_{1}, \ldots, x_{n}, y_{1}, \ldots, y_{n}$. The form $\omega_{0}=\sum_{i=1}^{n} d x_{i} \wedge d y_{i}$ is symplectic.

Example 1.An almost complex symplectic manifold is standard Euclidean space $\left(\mathbb{R}^{2 n}, \omega_{0}\right)$ with its standard almost complex structure $J_{0}$ obtained from the usual identification with $\mathbb{C}_{n}$. Thus, one sets $z_{j}=x_{2 j-1}+i x_{2 j}$ for $j=1, \ldots, n$ and defines $J_{0}$ by $J_{0}\left(\partial_{2 j-1}\right)=\partial_{2 j}, \quad J_{0}\left(\partial_{2 j}\right)=-\partial_{2 j-1}$ where $\partial_{j}=\partial / \partial x_{j}$ is the standard basis of $T_{x} \mathbb{R}^{2 n}[18]$.

Definition 8. Let $M$ be a differentiable manifold of dimension $2 n$, and suppose $J$ is a differentiable vector bundle isomorphism $J: T M \rightarrow T M$ such that $J_{x}: T_{x} M \rightarrow T_{x} M$ is a complex structure for $T_{x} M$, i.e. $J^{2}=-I$ where $I$ is the identity (unit) operator on $V$. Then $J$ is called an almost-complex structure for the differentiable manifold M. A manifold with a fixed almost complex structure is called an almost complex manifold [15].

\section{Gauge theory and conformal Weyl geometry}

Today, the gauge principle is arguably the most powerful concept in all of modern physics. This gauge principle underlies all of the Yang-Mills theories that it is a key component in string theory and its more recent variant, $M$ theory. The approach for studying conformal field theories is somewhat different from the usual approach for quantum and electromagnetic field theories. Conformal maps can be defined between domains in higher dimensional Euclidean spaces and on a Riemann or semi-Riemann manifold.

H. Weyl made many fundamental and important contributions to physics and he is most famous for his 1929 discovery of quantum-mechanical phase invariance. Phase invariance known more properly as gauge invariance that it is a symmetry and underlies all modern quantum theories. Also, Weyl tensor can be deduced by simply demanding that it be invariant with respect to this transformation. Weyl, using this gauge principle, was able to derive all of electrodynamics from a generalized Einstein-Maxwell Lagrangian. 
Definition 9. A conformal map or transformations is a function which preserves angles [19].

Definition 10. A conformal manifold is a differentiable manifold equipped with an equivalence class of (pseudo) Riemann metric tensors, in which two metrics $g_{2}$ and $g_{1}$ are equivalent if and only if

$$
g_{2}=\Psi^{2} g_{1}
$$

where $\Psi>0$ is a smooth positive function. An equivalence class of such metrics is known as a conformal metric or conformal class and a manifold with a conformal structure (2) is called a conformal manifold [19].

Definition 11. A change in given by the global gauge transformation

$$
\Psi(x) \rightarrow e^{i \lambda} \Psi(x)
$$

where $\lambda$ is an arbitrary constant, would have absolutely no effect on the Lagrangian [20].

Definition 12. Action Lagrangians are invariant with respect to the replacement

$$
\Psi(x) \rightarrow e^{i \lambda(x)} \Psi(x)
$$

can be made without changing anything essential. Where $\Psi$ is a wave function and $\lambda$ is an arbitrary function of space and time. (4) is called a local gauge transformation. Weyl's gauge theory sprang from an even earlier (1918) theory in which Weyl demanded that Einstein's theory of general relativity should be invariant with respect to the similar replacement

$$
g_{\mu v}(x) \rightarrow e^{\lambda(x)} g_{\mu v}(x)
$$

which we shall call a metric gauge transformation (5) and it has emerged effect of these transformations on Riemannian and non-Riemannian geometry [20].

Definition 13. Two Riemann metrics $g_{1}$ and $g_{2}$ on $M$ are said to be conformally equivalent iff there exists a smooth function $f: M \rightarrow \mathbb{R}$ with

$$
g_{2}=e^{f} g_{1}
$$

In this case, $g_{1} \sim g_{2}[21]$.

Definition 14. A pair $(M, G)$, a conformal structure on $M$ is an equivalence class $G$ of Riemann metrics on $M$, is called a conformal structure.

Theorem 1. Let $\nabla$ be a connection on $M$ and $g \in G$ a fixed metric. $\nabla$ is compatible with $(M, G) \Longleftrightarrow$ there exists a 1-form $\omega$ with $\nabla_{X} g+\omega(X) g=0[22]$.

Definition 15. A compatible torsion-free connection is called a Weyl connection. The triple $(M, G, \nabla)$ is a Weyl structure. A Weyl manifold is a conformal manifold equipped with a torsion free connection preserving the conformal structure, called a Weyl connection [21].

Definition 16. Consider a triple $(M, g, \nabla)$ where $g$ is a pseudo Riemannian metric on a smooth $n$ dimensional manifold $M$ and where $\nabla$ is a torsion free connection on the tangent bundle TM of M. We suppose $n \geq 2$ henceforth. We say that $(M, g, \nabla)$ is a Weyl manifold if the following identity is satisfied: $\nabla g=-2 \phi \otimes g$ for some $\phi \in \mathbb{C}^{\infty}\left(T^{*} M\right)$. This notion is conformally invariant. If $(M, g, \nabla)$ is a Weyl manifold, then $\left(M, e^{2 f} g, \nabla\right)$ is again a Weyl manifold where $\phi:=\phi-d f$. The simultaneous transformation of the pair $(g, \phi)$ is called a gauge transformation, properties of the Weyl geometry that are invariant under gauge transformations are called gauge invariants [23]. 
Theorem 2. To each metric $g \in G$ and 1 -form $\omega$, there corresponds a unique Weyl connection $\nabla$ satisfying $\nabla_{X} g+\omega(X) g=$ 0. Here, $\nabla$ is given by the equation [22].

$$
g\left(\nabla_{X} Y, Z\right)=\frac{1}{2}\left\{\begin{array}{l}
X(g(Y, Z))+\omega(X) g(Y, Z)-g([X, Z], Y)+Y(g(X, Z)) \\
+\omega(Y) g(Z, X)-g([Y, X], Z)-Z(g(X, Y))-\omega(Z) g(X, Y)-g([Z, Y], X)
\end{array}\right\}
$$

Definitions 17. Define a function $F:\{1$-forms on $M\} \times G \rightarrow\{$ Weyl connections $\}$ by $F(g, \omega)=\nabla$, where $\nabla$ is the connection guaranteed by Theorem 2 . We say that $\nabla$ corresponds to $(g, \omega)$ [22].

Proposition 2. $F$ is surjective.

Proof. $F$ is surjective by Theorem 1 . In fact, Theorem 1 . shows that given a compatible, torsion-free connection $\nabla$, then for every $g \in G$, there exists a 1 -form $\omega$ with $F(g, \omega)=\nabla$.

Proposition 3. $F(g, \omega)=F\left(e^{f} g, \eta\right)$ iff $\eta=\omega-d f$. So

$$
F\left(e^{f} g\right)=F(g)-d f
$$

where $G$ is a conformal structure. Note that a Riemann metric $g$ and a one-form $\omega$ determine a Weyl structure, namely $F: G \rightarrow \wedge^{1} M$ where $G$ is the equivalence class of $g$ and $F\left(e^{f} g\right)=\omega-d f[21,22]$.

Proof. Suppose $F(g, \omega)=F\left(e^{f} g, \eta\right)=\nabla$. We have

$$
0=\nabla_{X}\left(e^{f} g\right)+\eta(X) e^{f} g=X\left(e^{f}\right) g+e^{f} \nabla_{X} g+\eta(X) e^{f} g=d f(X) e^{f} g+e^{f} \nabla_{X} g+\eta(X) e^{f} g .
$$

Therefore $\nabla_{X} g=-(d f(X)+\eta(X))$. On the other hand $\nabla_{X} g+\omega(X) g=0$ and $\omega=\eta+d f$. Conversely, suppose $\eta=$ $\omega-d f$. Set $\nabla=F(g, \omega)$. To show $\nabla=F\left(e^{f} g, \eta\right)$, it suffices, by the uniqueness of Theorem 2 , to show $\nabla_{X}\left(e^{f} g\right)+$ $\eta(X) e^{f} g=0$. Let's show the truth of this statement. $\nabla_{X}\left(e^{f} g\right)=e^{f} d f(X) g+e^{f} \nabla_{X} g$ and $\eta(X)=\omega(X)-d f(X)$.

$$
\begin{aligned}
& \nabla_{X}\left(e^{f} g\right)+\eta(X) e^{f} g=e^{f} d f(X) g+e^{f} \nabla_{X} g+(\omega(X)-d f(X)) e^{f} g \\
& \quad=e^{f} d f(X) g+e^{f} \nabla_{X} g+\omega(X) e^{f} g-d f(X) e^{f} g=e^{f}\left(\nabla_{X} g+\omega(X) g\right)=0 .
\end{aligned}
$$

Theorem 3. Let $(M, g)$ is conformally flat if for each point $x$ in $M$, there exists a neighborhood $U$ of $x$ and a smooth function $f$ defined on $U$ such that $\left(U, e^{2 f} g\right)$ is flat. The function $f$ need not be defined on all of $M$ [24].

Theorem 4. Let $m \geq 6$. If $(M, g, J, \nabla)$ is a (para)-Kähler-Weyl structure, then the associated Weyl structure is trivial, i.e. there is a conformally equivalent metric

$$
g_{1}=e^{2 f} g
$$

so that $\left(M, g_{1}, J\right)$ is (para)-Kähler and so that $\nabla=\nabla^{g_{1}}$ [25].

Definition 17. Weyl curvature tensor is a measure of the curvature of spacetime or a pseudo-Riemannian manifold. Like the Riemannian curvature tensor, the Weyl tensor expresses the tidal force that a object feels when moving along a geodesic [26].

Definition 18. Weyl transformation is a local rescaling of the metric tensor: $g_{a b}(x) \rightarrow e^{-2 \omega(x)} g_{a b}(x)$ which produces another metric in the same conformal class. A theory or an expression invariant under this transformation is called conformally invariant, or is said to possess Weyl symmetry. The Weyl symmetry is an important symmetry in conformal field theory [27]. 


\section{Properties of almost complex structure $J$}

Example 2. Let $O$ be an open subset of $\mathbb{R}^{4}$. Let $a, b, c \in C^{\infty}(O)$ be smooth function on $O$. We set $M_{a, b, c}:=\left(O, g_{a, b, c}\right)$ where

$$
\begin{aligned}
& g_{a, b, c}:=2\left(d x_{1} \circ d x_{3}+d x_{2} \circ d x_{4}\right)+a\left(x_{1}, x_{2}, x_{3}, x_{4}\right) d x_{3} \circ d x_{3} \\
& \quad+b\left(x_{1}, x_{2}, x_{3}, x_{4}\right) d x_{34} \circ d x_{4}+2 c\left(x_{1}, x_{2}, x_{3}, x_{4}\right) d x_{3} \circ d x_{4} .
\end{aligned}
$$

Example 3. Let $M_{a, b, c}:=\left(O, g_{a, b, c}\right)$ be the pseudo-Riemannian manifold of (12) and we take the induced orthonormal basis;

$$
\begin{gathered}
e_{1}=\frac{1}{2}(1-a) \partial_{x_{1}}+\partial_{x_{3}}, e_{2}=-c \partial_{x_{2}}+\frac{1}{2}(1-b) \partial_{x_{4}} \\
e_{3}=-\frac{1}{2}(1+a) \partial_{x_{1}}+\partial_{x_{3}} e_{4}=-c \partial_{x_{2}}-\frac{1}{2}(1+b) \partial_{x_{4}} .
\end{gathered}
$$

We shall let $C_{a, b, c}:=\left(O, g_{a, b, c}, J\right)$ where $J$ is the proper almost complex structure defined by $J=e_{2} \otimes e^{1}-e_{1} \otimes e^{2}+e_{4} \otimes$ $e^{3}-e_{3} \otimes e^{4}$. Thus, the following expression is obtained: $J: e_{1}=e_{2}, J: e_{2}=-e_{1}, J: e_{3}=e_{4}, J: e_{4}=-e_{3}$. The map $J$ induces a positive $\frac{\pi}{2}$-rotation on the degenerate parallel field $D:=\operatorname{Span}\left\{\partial x_{1}, \partial x_{2}\right\}$ :

$$
\begin{aligned}
& J \partial_{x_{1}}=\partial_{x_{2}}, \quad J \partial_{x_{3}}=-c \partial_{x_{1}}+\frac{1}{2}(a-b) \partial_{x_{2}}+\partial_{x_{4}} \\
& J \partial_{x_{2}}=-\partial_{x_{1}}, J \partial_{x_{4}}=\frac{1}{2}(a-b) \partial_{x_{1}}+c \partial_{x_{2}}-\partial_{x_{3}} .
\end{aligned}
$$

The above structures were taken from [14,28]. The following result shows that the class of isotropic Kähler structures is larger than might at first sight be expected:

Theorem 5. Let $C_{a, b, c}:=O, g_{a, b, c}$ be as given in Example 2. $C_{a, b, c}$ is almost Kähler if and only if $a_{1}+b_{1}=0$ and $a_{2}+b_{2}=$ 0, (2) $C_{a, b, c}$ is Hermitian if and only if $a_{1}+b_{1}=2 c_{2}$ and $a_{2}-b_{2}=-2 c_{1}$, (3) $C_{a, b, c}$ is is Kähler if and only if $a_{1}=-b_{1}=c_{2}$ and $a_{2}=-b_{2}=-c_{1}[14]$.

Definition 19. In three dimensions, the vector from the origin to the point with Cartesian coordinates $(x, y, z)$ can be written as: $r=x \mathbf{i}+y \mathbf{j}+z \mathbf{k}=x\left(\frac{\partial}{\partial x}\right)+y\left(\frac{\partial}{\partial y}\right)+z\left(\frac{\partial}{\partial z}\right)$.

Proposition 4. We recommend transferring the functions of the Weyl conformal factor as follows. J holomorphic property preservation is proposed using Theorems 4 and Definition 19 as follows.

$$
\begin{aligned}
& \text { (1) } J \frac{\partial}{\partial_{x_{1}}}=e^{2 f} \frac{\partial}{\partial_{x_{2}}}, \quad \text { (3) } J \frac{\partial}{\partial_{x_{3}}}=-c e^{-2 f} \frac{\partial}{\partial_{x_{1}}}+\frac{1}{2}(a-b) e^{2 f} \frac{\partial}{\partial_{x_{2}}}+e^{2 f} \frac{\partial}{\partial_{x_{4}}} \text {, } \\
& \text { (2) } J \frac{\partial}{\partial_{x_{2}}}=-e^{-2 f} \frac{\partial}{\partial_{x_{1}}} \text {, (4) } J \frac{\partial}{\partial_{x_{4}}}=\frac{1}{2}(a-b) e^{-2 f} \frac{\partial}{\partial_{x_{1}}}+c e^{-2 f} \frac{\partial}{\partial_{x_{2}}}-e^{-2 f} \frac{\partial}{\partial_{x_{3}}} \text {, }
\end{aligned}
$$

Proof.

$$
\begin{aligned}
& \text { (1) } J^{2} \frac{\partial}{\partial_{x_{1}}}=e^{2 f} J \frac{\partial}{\partial_{x 2}}=-\frac{\partial}{\partial_{x_{1}}}, \\
& \text { (2) } J^{2} \frac{\partial}{\partial_{x_{2}}}=-e^{-2 f} J \frac{\partial}{\partial_{x_{1}}}=-\frac{\partial}{\partial_{x_{2}}}, \\
& \text { (3) } J^{2} \frac{\partial^{2}}{\partial_{x_{3}}}=-c e^{-2 f} J \frac{\partial}{\partial_{x_{1}}}+\frac{1}{2}(a-b) e^{2 f} J \frac{\partial}{\partial_{x_{2}}}+e^{2 f} J \frac{\partial}{\partial_{x_{4}}}=-\frac{\partial}{\partial_{x_{3}}}, \\
& \text { (4) } J^{2} \frac{\partial^{2}}{\partial_{x_{4}}}=\frac{1}{2}(a-b) e^{-2 f} J \frac{\partial}{\partial_{x_{1}}}+c e^{-2 f} J \frac{\partial^{2}}{\partial_{x_{2}}}-e^{-2 f} J \frac{\partial}{\partial_{x_{3}}}=-\frac{\partial}{\partial_{x_{4}}} .
\end{aligned}
$$

As seen above; holomorphic structures $\left(J^{2} \frac{\partial}{\partial_{x_{i}}}=-\frac{\partial}{\partial_{x_{i}}}\right.$ or $\left.J^{2}=-I\right)$ are complex.

\section{Euler-Lagrange dynamics equations}

The Euler-Lagrange equation, Euler's equation or Lagrange's equation is a second-order partial differential equation whose solutions are the functions for which a given functional is stationary.

Lemma 1. The closed 2-form $(\omega)$ on a vector field $(\xi)$ and 1 -form reduction function $\left(i_{\xi}\right)$ on the phase space defined of a mechanical system $\left(i_{\xi} \omega\right)$ is equal to the differential of the energy function 1-form $(d E)$ of the Hamiltonian and the Lagrangian mechanical systems [1]. 
Definition 20. Let $M$ be an n-dimensional manifold and TM its tangent bundle with canonical projection $\tau_{M}: T M \rightarrow M$. $T M$ is called the phase space of velocities of the base manifold M. Let $L: T M \rightarrow R$ be a differentiable function on TM called the Lagrangian function. Here, $L=T-V$ such that $T$ is the kinetic energy and $V$ is the potential energy of a mechanical system. In the problem of a mass on the end of a spring, $T=m \dot{x}^{2} / 2$ and $V=k x^{2} / 2$. We consider the closed 2-form and base space $(J)$ on TM given by $\Phi_{L}=-d \mathbf{d}_{J} L=-d(J(\mathbf{d}))$. Consider the equation

$$
i_{\xi} \Phi_{L}=d E_{L}
$$

Where $i_{\xi}$ is reduction function and $i_{\xi} \Phi_{L}=\Phi_{L}(\xi)$ is defined in the form. Then $\xi$ is a vector field, we shall see that (17) under a certain condition on $\xi$ is the intrinsical expression of the Euler-Lagrange equations of motion. This equation (17) is named as Lagrange dynamical equation [15].

Definition 21. We shall see that for motion in a potential, $E_{L}=V L-L$ is an energy function and $V=J \xi$ a Liouville vector field. Here $d E_{L}$ denotes the differential of $E$. The triple $\left(T M, \Phi_{L}, \xi\right)$ is known as Lagrangian system on the tangent bundle TM. If it is continued the operations on (17) for any coordinate system then infinite dimension Lagrange's equation is obtained the form below. The equations of motion in Lagrangian mechanics are the Lagrange equations of the second kind, also known as the Euler-Lagrange equations [2].

$$
\frac{\partial}{\partial t}\left(\frac{\partial L}{\partial \dot{x}}\right)-\frac{\partial L}{\partial x}=0
$$

Definition 22. Newton's second law of motion for the mechanical problem is $F=m a$. Where, the vector sum of the external forces $F$ on an object is equal to the mass $m$ of that object multiplied by the acceleration vector a of the object.

Proposition 5. We have $\partial L / \partial \dot{x}=m x$ and $\partial L / \partial x=-k x$, so eq. (18) gives $m \ddot{x}=-k x$. The Euler-Lagrange equation, eq. (18), gives $m \ddot{x}=-d V / d x$. In a four-dimensional setup written in terms of Cartesian coordinates, the potential takes the form $V\left(x_{1}, x_{2}, x_{3}, x_{4}\right)$, so the Lagrangian is $L=\frac{1}{2} m_{i}\left(\dot{x}_{1}{ }^{2}+\dot{x}_{2}^{2}+\dot{x}_{3}^{2}+\dot{x}_{4}^{2}\right)-V\left(x_{1}, x_{2}, x_{3}, x_{4}\right)$. So, the three Euler-Lagrange equations may be combined into the vector statement $m \ddot{x}=-\nabla V$.

\section{Conformal Weyl-Euler-Lagrange equations}

In this section we, using (17), will introduced the movement equation on Walker manifold. Let $M_{4}$ be a Walker manifold and $\left\{x_{1}, x_{2}, x_{3}, x_{4}\right\}$ be its coordinate. Let $\xi$ be a the semispray vector field determined by

$$
\xi=\sum_{i=1}^{4} X^{i} \frac{\partial}{\partial_{x_{i}}}
$$

Also, $X^{i}=\dot{x}_{i}$ the dot indicates the derivative with respect to time $t$. Euler-Lagrange equations are obtained by using. By means of the proper almost complex structure $J$ given by (15), the vector field is defined by

$$
\begin{aligned}
& V=J(\xi)=X^{1} e^{2 f} \frac{\partial}{\partial_{x_{2}}}-X^{2} e^{-2 f} \frac{\partial}{\partial_{x_{1}}}+X^{3}\left(-c e^{-2 f} \frac{\partial}{\partial_{x_{1}}}+\frac{1}{2}(a-b) e^{2 f} \frac{\partial}{\partial_{x_{2}}}+e^{2 f} \frac{\partial}{\partial_{x_{4}}}\right) \\
& +X^{4}\left(\frac{1}{2}(a-b) e^{-2 f} \frac{\partial}{\partial_{x_{1}}}+c e^{-2 f} \frac{\partial}{\partial_{x_{2}}}-e^{-2 f} \frac{\partial}{\partial_{x_{3}}}\right),
\end{aligned}
$$

which is named Liouville vector field on the Walker manifold $M_{4}$. The maps given by $T, P: M_{4} \rightarrow \mathbb{R}$ such that $T=$ $m_{i}\left(\dot{x}_{1}{ }^{2}+\dot{x}_{2}^{2}+\dot{x}_{3}^{2}+\dot{x}_{4}^{2}\right) / 2, V=m_{i} g h$ are said to be the kinetic energy and the potential energy of the system, respectively. Here $m_{i}, g$ and $h$ stand for mass of a mechanical system having $m$ particles, the gravity acceleration and distance to the origin of a mechanical system on Walker manifold $M_{4}$, respectively. Then $L: M_{4} \rightarrow \mathbb{R}$ is a map that satisfies the conditions; (i) $L=T-V$ is a Lagrangian function, (ii) the function determined by $E_{L}=V L-L$, is energy function. The function $i_{J}$ induced by $J$ and denoted by

$$
i_{J} \omega\left(X_{1}, X_{2}, \ldots, X_{r}\right)=\sum_{i=1}^{r} J\left(X_{1}, \ldots, J X_{i}, \ldots, X_{r}\right)
$$


is called vertical derivation, where $\omega \in \wedge^{r} M_{4}, X_{i} \in \chi\left(M_{4}\right)$. The vertical differentiation $d_{J}$ is given by $\mathbf{d}_{J}=\left[i_{J}, d\right]=$ $i_{J} d-d i_{J}$, where $d$ is the usual exterior derivation. For the almost complex structure $J$ given by (15), the form on Walker manifold $M_{4}$ is the closed 2-form determined by $\Phi_{L}=-d \mathbf{d}_{J} L$ such that $d_{J}: \mathscr{F}\left(M_{4}\right) \rightarrow \wedge^{1} M_{4}$,

$$
\begin{aligned}
& \mathbf{d}=\sum_{i=1}^{4} \frac{\partial}{\partial_{x_{i}}} d x_{i}, \text { and } \\
& \begin{aligned}
\mathbf{d}_{J}=J(\mathbf{d})= & e^{2 f} \frac{\partial}{\partial_{x_{2}}} d x_{1}-e^{-2 f} \frac{\partial}{\partial_{x_{1}}} d x_{2}+\left(-c e^{-2 f} \frac{\partial}{\partial_{x_{1}}}+\frac{1}{2}(a-b) e^{2 f} \frac{\partial}{\partial_{x_{2}}}+e^{2 f} \frac{\partial}{\partial_{x_{4}}}\right) d x_{3} \\
& +\left(\frac{1}{2}(a-b) e^{-2 f} \frac{\partial}{\partial_{x_{1}}}+c e^{-2 f} \frac{\partial}{\partial_{x_{2}}}-e^{-2 f} \frac{\partial}{\partial_{x_{3}}}\right) d x_{4} .
\end{aligned}
\end{aligned}
$$

Now, we calculate the first part $\left(i_{\xi} \Phi_{L}\right)$ of (17). Through a direct computation using (22), the closed 2-form $\Phi_{L}=-d(J(\mathbf{d}))$ is seen to be as follows:

$$
\Phi_{L}=\sum_{i=1}^{4}\left[\begin{array}{c}
\left(e^{2 f} \frac{\partial^{2} L}{\partial_{x_{i}} \partial_{x_{2}}}+2 e^{2 f} \frac{\partial f}{\partial_{x_{i}}} \frac{\partial L}{\partial_{x_{2}}}\right) d x_{1} \wedge d x_{i}+\left(-e^{-2 f} \frac{\partial^{2} L}{\partial_{x_{i}} \partial_{x_{1}}}+2 e^{-2 f} \frac{\partial f}{\partial_{x_{i}}} \frac{\partial L}{\partial_{x_{1}}}\right) d x_{2} \wedge d x_{i} \\
+\left(\begin{array}{c}
-c e^{-2 f} \frac{\partial^{2} L}{\partial_{x_{i}} \partial_{x_{1}}}+2 c e^{-2 f} \frac{\partial f}{\partial_{x_{i}}} \frac{\partial L}{\partial_{x_{1}}}+\frac{1}{2}(a-b) e^{2 f} \frac{\partial^{2} L}{\partial_{x_{i}} \partial_{x_{2}}} \\
+2 \frac{1}{2}(a-b) 2 e^{2 f} \frac{\partial f}{\partial_{x_{i}}} \frac{\partial L}{\partial_{x_{2}}}+e^{2 f} \frac{\partial^{2} L}{\partial_{x_{i}} \partial_{x_{4}}}+2 e^{2 f} \frac{\partial f}{\partial_{x_{i}}} \frac{\partial L}{\partial_{x_{4}}}
\end{array}\right) d x_{3} \wedge d x_{i} \\
+\left(\begin{array}{c}
\frac{1}{2}(a-b) e^{-2 f} \frac{\partial^{2} L}{\partial_{x_{i}} \partial_{x_{1}}}-2 \frac{1}{2}(a-b) e^{-2 f} \frac{\partial f}{\partial_{x_{i}}} \frac{\partial L}{\partial x_{1}}+c e^{-2 f} \frac{\partial^{2} L}{\partial_{x_{i}} \partial_{x_{2}}} \\
-2 c e^{-2 f} \frac{\partial f}{\partial_{x_{i}}} \frac{\partial L}{\partial_{x_{2}}}-e^{-2 f} \frac{\partial^{2} L}{\partial_{x_{i}} \partial_{x_{3}}}+2 e^{-2 f} \frac{\partial f}{\partial_{x_{i}}} \frac{\partial L}{\partial_{x_{3}}}
\end{array}\right) d x_{4} \wedge d x_{i}
\end{array}\right] .
$$

We obtain, $\xi$ vector based processing on (23), the following statement $\Phi_{L}(\xi)$;

$$
\begin{aligned}
& -X^{1}\left(e^{2 f} \frac{\partial^{2} L}{\partial_{x_{1}} \partial_{x_{2}}}+2 e^{2 f} \frac{\partial f}{\partial_{x_{1}}} \frac{\partial L}{\partial_{x_{2}}}\right) d x_{1}-X^{1}\left(-e^{-2 f} \frac{\partial^{2} L}{\partial_{x_{1}} \partial_{x_{1}}}+2 e^{-2 f} \frac{\partial f}{\partial_{x_{1}}} \frac{\partial L}{\partial_{x_{1}}}\right) d x_{2} \\
& -X^{1}\left(\begin{array}{c}
-c e^{-2 f} \frac{\partial^{2} L}{\partial_{x_{1}} \partial_{x_{1}}}+2 c e^{-2 f} \frac{\partial f}{\partial_{x_{1}}} \frac{\partial L}{\partial x_{1}}+\frac{1}{2}(a-b) e^{2 f} \frac{\partial^{2} L}{\partial_{x_{1}} \partial_{x_{2}}} \\
+2 \frac{1}{2}(a-b) e^{2 f} \frac{\partial f}{\partial_{x_{1}}} \frac{\partial L}{\partial_{x_{2}}}+e^{2 f} \frac{\partial^{2} L}{\partial_{x_{1}} \partial_{x_{4}}}+2 e^{2 f} \frac{\partial f}{\partial_{x_{1}}} \frac{\partial L}{\partial_{x_{4}}}
\end{array}\right) d x_{3} \\
& -X^{1}\left(\begin{array}{c}
\frac{1}{2}(a-b) e^{-2 f} \frac{\partial^{2} L}{\partial_{x_{1}} \partial_{x_{1}}}-2 \frac{1}{2}(a-b) e^{-2 f} \frac{\partial f}{\partial_{x_{1}}} \frac{\partial L}{\partial x_{1}}+c e^{-2 f} \frac{\partial^{2} L}{\partial_{x_{1}} \partial_{x_{2}}} \\
-2 c e^{-2 f} \frac{\partial f}{\partial_{x_{1}}} \frac{\partial L}{\partial_{x_{2}}}-e^{-2 f} \frac{\partial^{2} L}{\partial_{x_{1}} \partial_{x_{3}}}+2 e^{-2 f} \frac{\partial f}{\partial_{x_{1}}} \frac{\partial L}{\partial_{x_{3}}}
\end{array}\right) d x_{4} \\
& -X^{2}\left(\begin{array}{c}
-c e^{-2 f} \frac{\partial^{2} L}{\partial_{x_{2}} \partial_{x_{1}}}+2 c e^{-2 f} \frac{\partial \Psi}{\partial_{x_{2}}} \frac{\partial L}{\partial_{x_{1}}}+\frac{1}{2}(a-b) e^{2 f} \frac{\partial^{2} L}{\partial_{x_{2}} \partial_{x_{2}}} \\
+2 \frac{1}{2}(a-b) e^{2 f} \frac{\partial f}{\partial_{x_{2}}} \frac{\partial L}{\partial_{x_{2}}}+e^{2 f} \frac{\partial^{2} L}{\partial_{x_{2}} \partial_{x_{4}}}+2 e^{2 f} \frac{\partial f}{\partial_{x 2}} \frac{\partial L}{\partial_{x_{4}}}
\end{array}\right) d x_{3} \\
& -X^{2}\left(\begin{array}{c}
\frac{1}{2}(a-b) e^{-2 f} \frac{\partial^{2} L}{\partial_{x_{2}} \partial_{x_{1}}}-2 \frac{1}{2}(a-b) e^{-2 f} \frac{\partial f}{\partial_{x_{2}}} \frac{\partial L}{\partial_{x_{1}}} \\
+c e^{-2 f} \frac{\partial^{2} L}{\partial_{x_{2}} \partial_{x_{2}}}-2 c e^{-2 f} \frac{\partial f}{\partial_{x_{2}}} \frac{\partial L}{\partial_{x_{2}}}-e^{-2 f} \frac{\partial^{2} L}{\partial_{x 2} \partial_{x_{3}}}+2 e^{-2 f} \frac{\partial f}{\partial_{x 2}} \frac{\partial L}{\partial_{x_{3}}}
\end{array}\right) d x_{4} \\
& -X^{3}\left(e^{2 f} \frac{\partial^{2} L}{\partial_{x_{3}} \partial_{x_{2}}}+2 e^{2 f} \frac{\partial f}{\partial_{x_{3}}} \frac{\partial L}{\partial_{x_{2}}}\right) d x_{1}-X^{3}\left(-e^{-2 f} \frac{\partial^{2} L}{\partial_{x_{3}} \partial_{x_{1}}}+2 e^{-2 f} \frac{\partial f}{\partial_{x_{3}}} \frac{\partial L}{\partial_{x_{1}}}\right) d x_{2} \\
& -X^{3}\left(\begin{array}{c}
-c e^{-2 f} \frac{\partial^{2} L}{\partial_{x_{3}} \partial_{x_{1}}}+2 c e^{-2 f} \frac{\partial f}{\partial_{x_{3}}} \frac{\partial L}{\partial_{x_{1}}}+\frac{1}{2}(a-b) e^{2 f} \frac{\partial^{2} L}{\partial_{x_{3}} \partial_{x_{2}}} \\
+2 \frac{1}{2}(a-b) e^{2 f} \frac{\partial f}{\partial_{x_{3}}} \frac{\partial L}{\partial_{x_{2}}}+e^{2 f} \frac{\partial^{2} L}{\partial_{x_{3}} \partial_{x_{4}}}+2 e^{2 f} \frac{\partial f}{\partial_{x_{3}}} \frac{\partial L}{\partial_{x_{4}}}
\end{array}\right) d x_{3} \\
& -X^{3}\left(\begin{array}{c}
\frac{1}{2}(a-b) e^{-2 f} \frac{\partial^{2} L}{\partial_{x_{3}} \partial_{x_{1}}}-2 \frac{1}{2}(a-b) e^{-2 f} \frac{\partial f}{\partial_{x_{3}}} \frac{\partial L}{\partial_{x_{1}}}+c e^{-2 f} \frac{\partial^{2} L}{\partial_{x_{3}} \partial_{x_{2}}} \\
-2 c e^{-2 f} \frac{\partial f}{\partial_{x_{3}}} \frac{\partial L}{\partial_{x_{2}}}-e^{-2 f} \frac{\partial^{2} L}{\partial_{x_{3}} \partial_{x_{3}}}+2 e^{-2 f} \frac{\partial f}{\partial_{x_{3}}} \frac{\partial L}{\partial_{x_{3}}}
\end{array}\right) d x_{4} \\
& -X^{4}\left(e^{2 f} \frac{\partial^{2} L}{\partial_{x_{4}} \partial_{x_{2}}}+2 e^{2 f} \frac{\partial f}{\partial_{x_{4}}} \frac{\partial L}{\partial_{x_{2}}}\right) d x_{1}-X^{4}\left(-e^{-2 f} \frac{\partial^{2} L}{\partial_{x_{4}} \partial_{x_{1}}}+2 e^{-2 f} \frac{\partial f}{\partial_{x_{4}}} \frac{\partial L}{\partial_{x_{1}}}\right) d x_{2} \\
& -X^{4}\left(\begin{array}{c}
-c e^{-2 f} \frac{\partial^{2} L}{\partial_{x_{4}} \partial_{x_{1}}}+2 c e^{-2 f} \frac{\partial f}{\partial_{x 4}} \frac{\partial L}{\partial_{x_{1}}}+\frac{1}{2}(a-b) e^{2 f} \frac{\partial^{2} L}{\partial_{x 4} \partial_{x_{2}}} \\
+2 \frac{1}{2}(a-b) e^{2 f} \frac{\partial f}{\partial_{x_{4}}} \frac{\partial L}{\partial_{x_{2}}}+e^{2 f} \frac{\partial^{2} L}{\partial_{x_{4}} \partial_{x_{4}}}+2 e^{2 f} \frac{\partial f}{\partial_{x_{4}}} \frac{\partial L}{\partial_{x_{4}}}
\end{array}\right) d x_{3} \\
& -X^{4}\left(\begin{array}{c}
\frac{1}{2}(a-b) e^{-2 f} \frac{\partial^{2} L}{\partial_{x 4} \partial_{x_{1}}}-2 \frac{1}{2}(a-b) e^{-2 f} \frac{\partial f}{\partial_{x_{4}}} \frac{\partial L}{\partial_{x_{1}}}+c e^{-2 f} \frac{\partial^{2} L}{\partial_{x_{4}} \partial_{x_{2}}} \\
-2 c e^{-2 f} \frac{\partial f}{\partial_{x 4}} \frac{\partial L}{\partial_{x_{2}}}-e^{-2 f} \frac{\partial^{2} L}{\partial_{x_{4}} \partial_{x_{3}}}+2 e^{-2 f} \frac{\partial f}{\partial_{x_{4}}} \frac{\partial L}{\partial_{x_{3}}}
\end{array}\right) d x_{4} .
\end{aligned}
$$


Then the energy function $E_{L}$ is found as follows:

$$
\begin{aligned}
& E_{L}=V(L)-L=X^{1} e^{2 f} \frac{\partial L}{\partial_{x_{2}}}-X^{2} e^{-2 f} \frac{\partial L}{\partial_{x_{1}}}+X^{3}\left(-c e^{-2 f} \frac{\partial L}{\partial_{x_{1}}}+\frac{1}{2}(a-b) e^{2 f} \frac{\partial L}{\partial_{x_{2}}}+e^{2 f} \frac{\partial L}{\partial_{x_{4}}}\right) \\
& +X^{4}\left(\frac{1}{2}(a-b) e^{-2 f} \frac{\partial L}{\partial_{x_{1}}}+c e^{-2 f} \frac{\partial L}{\partial_{x_{2}}}-e^{-2 f} \frac{\partial L}{\partial_{x_{3}}}\right)-L .
\end{aligned}
$$

Now, we calculate the second part of (17). Thus, the differential energy function is as follows:

$$
d E_{L}=\sum_{i=1}^{4}\left[\begin{array}{c}
X^{1}\left[e^{2 f} \frac{\partial^{2} L}{\partial_{x_{i}} \partial_{x_{2}}} d x_{i}+2 e^{2 f} \frac{\partial f}{\partial_{x_{i}}} \frac{\partial L}{\partial_{x_{2}}} d x_{i}\right]-X^{2}\left[e^{-2 f} \frac{\partial^{2} L}{\partial_{x_{i}} \partial_{x_{1}}} d x_{i}-2 e^{-2 f} \frac{\partial f}{\partial_{x_{i}}} \frac{\partial L}{\partial x_{1}} d x_{i}\right] \\
+X^{3}\left[\begin{array}{c}
-c e^{-2 f} \frac{\partial^{2} L}{\partial_{x_{i}} \partial_{x_{1}}} d x_{i}+2 c e^{-2 f} \frac{\partial f}{\partial_{x_{i}}} \frac{\partial L}{\partial x_{1}} d x_{i}+\frac{1}{2}(a-b) e^{2 f} \frac{\partial^{2} L}{\partial_{x_{i}} \partial_{x_{2}}} d x_{i} \\
+\frac{1}{2}(a-b) 2 e^{2 f} \frac{\partial f}{\partial_{x_{i}}} \frac{\partial L}{\partial_{x_{2}}} d x_{i}+e^{2 f} \frac{\partial^{2} L}{\partial_{x_{i}} \partial_{x_{4}}} d x_{i}+2 e^{2 f} \frac{\partial f}{\partial_{x_{i}}} \frac{\partial L}{\partial_{x_{4}}} d x_{i}
\end{array}\right] \\
+X^{4}\left[\begin{array}{c}
\frac{1}{2}(a-b) e^{-2 f} \frac{\partial^{2} L}{\partial_{x_{i}} \partial_{x_{1}}} d x_{i}-\frac{1}{2}(a-b) 2 e^{-2 f} \frac{\partial f}{\partial_{x_{i}}} \frac{\partial L}{\partial_{x_{1}}} d x_{i}+c e^{-2 f} \frac{\partial^{2} L}{\partial_{x_{i}} \partial_{x_{2}}} d x_{i} \\
-c 2 e^{-2 f} \frac{\partial f}{\partial_{x_{i}}} \frac{\partial L}{\partial_{x_{2}}} d x_{i}-e^{-2 f} \frac{\partial^{2} L}{\partial_{x_{i}} \partial_{x 3}} d x_{i}+2 e^{-2 f} \frac{\partial f}{\partial_{x_{i}}} \frac{\partial L}{\partial_{x_{3}}} d x_{i}
\end{array}\right]-\frac{\partial L}{\partial_{x_{i}}} d x_{i}
\end{array}\right] .
$$

Definition 23. Suppose that $\xi: \mathbb{R} \rightarrow M_{4}$ is a vector field: that is, a vector-valued function with Cartesian coordinates $\left(\xi_{1}, \ldots, \xi_{n}\right)$; and $\mathbf{x}(t)$ a parametric curve with Cartesian coordinates $\left(x_{1}(t), \ldots, x_{n}(t)\right)$. Then $\mathbf{x}(t)$ is an integral curve of $\xi$ if it is a solution of the following autonomous system of ordinary differential equations: $\frac{d x_{1}}{d t}=\xi_{1}\left(x_{1}, \ldots, x_{n}\right), \ldots, \frac{d x_{n}}{d t}=$ $\xi_{n}\left(x_{1}, \ldots, x_{n}\right)$. Such a system may be written as a single vector equation

$$
\xi(\mathbf{x}(t))=\mathbf{x}^{\prime}(t)=\frac{\partial}{\partial t}(\mathbf{x}(t))
$$

According to Theorem 5, using $(24,25)$ and(27), then we find the following first equations:

$$
\begin{aligned}
& -X^{1}\left(e^{2 f} \frac{\partial^{2} L}{\partial_{x_{1}} \partial_{x_{2}}}+2 e^{2 f} \frac{\partial f}{\partial_{x_{1}}} \frac{\partial L}{\partial_{x_{2}}}\right) d x_{1}-X^{2}\left(e^{2 f} \frac{\partial^{2} L}{\partial_{x_{2}} \partial_{x_{2}}}+2 e^{2 f} \frac{\partial f}{\partial_{x_{2}}} \frac{\partial L}{\partial_{x_{2}}}\right) d x_{1} \\
& -X^{3}\left(e^{2 f} \frac{\partial^{2} L}{\partial_{x_{3}} \partial_{x_{2}}}+2 e^{2 f} \frac{\partial f}{\partial_{x_{3}}} \frac{\partial L}{\partial_{x_{2}}}\right) d x_{1}-X^{4}\left(e^{2 f} \frac{\partial^{2} L}{\partial_{x_{1}} \partial_{x_{2}}}+2 e^{2 f} \frac{\partial f}{\partial_{x_{4}}} \frac{\partial L}{\partial_{x_{2}}}\right) d x_{1}=-\frac{\partial L}{\partial_{x_{1}}} d x_{1}, \\
& -\left(X^{1} \frac{\partial}{\partial_{x_{1}}}+X^{2} \frac{\partial}{\partial_{x_{2}}}+X^{3} \frac{\partial}{\partial_{x_{3}}}+X^{4} \frac{\partial}{\partial_{x_{4}}}\right)\left(e^{2 f} \frac{\partial L}{\partial_{x_{2}}}\right)+\frac{\partial L}{\partial_{x_{1}}}=0, \\
& -\xi\left(e^{2 f} \frac{\partial L}{\partial x_{2}}\right)+\frac{\partial L}{\partial_{x_{1}}}=0, \\
& -\frac{\partial}{\partial t}\left(e^{2 f} \frac{\partial L}{\partial_{x_{2}}}\right)+\frac{\partial L}{\partial_{x_{1}}}=0 .
\end{aligned}
$$

Performed a similar procedure as in (29) for (17) are obtained equations below:

$$
\begin{aligned}
& \operatorname{dif} 1:-\frac{\partial}{\partial t}\left(e^{2 f} \frac{\partial L}{\partial_{x_{2}}}\right)+\frac{\partial L}{\partial_{x_{1}}}=0, \\
& \operatorname{dif} 2: \frac{\partial}{\partial t}\left(e^{-2 f} \frac{\partial L}{\partial_{x_{1}}}\right)+\frac{\partial L}{\partial_{x_{2}}}=0, \\
& \text { dif } 3: c \frac{\partial}{\partial t}\left(e^{-2 f} \frac{\partial L}{\partial_{x_{1}}}\right)-\frac{1}{2}(a-b) \frac{\partial}{\partial t}\left(e^{2 f} \frac{\partial L}{\partial_{x_{2}}}\right)-\frac{\partial}{\partial t}\left(e^{2 f} \frac{\partial L}{\partial_{x_{4}}}\right)+\frac{\partial L}{\partial_{x_{3}}}=0, \\
& \text { dif } 4:-\frac{1}{2}(a-b) \frac{\partial}{\partial t}\left(e^{-2 f} \frac{\partial L}{\partial_{x_{1}}}\right)-c \frac{\partial}{\partial t}\left(e^{-2 f} \frac{\partial L}{\partial_{x_{2}}}\right)+\frac{\partial}{\partial t}\left(e^{-2 f} \frac{\partial L}{\partial_{x 3}}\right)+\frac{\partial L}{\partial_{x 4}}=0 .
\end{aligned}
$$

The equations calculated in (30) are named conformal Weyl-Euler-Lagrange equations constructed on Walker manifold $M_{4}$ and thus the triple $\left(M_{4}, \Phi_{L}, \xi\right)$ is named a conformal Weyl-Euler-Lagrange mechanical system on Walker-Weyl manifold $\left(M_{4}, g, D, \nabla, J\right)$. 


\section{Weyl-Euler-Lagrangian equations for conservative dynamical systems}

Proposition 6. We choose $F=\mathbf{i}_{\xi}, g=\Phi_{L}$ and $f \rightarrow 2 f$ at (17) and we consider the equation $F\left(e^{f} g\right)=F(g)-d f$, we can write Weyl-Lagrangian dynamic equation as follows:

$$
\mathbf{i}_{\xi}\left(e^{2 f} \Phi_{L}\right)=\mathbf{i}_{\xi}\left(\Phi_{L}\right)-d(2 f)
$$

The second part (17), according to the law of conservation of energy [2], will not change for conservative dynamical systems and $\mathbf{i}_{\xi}\left(\Phi_{L}\right)=\Phi_{L}(\xi), \Phi_{L}(\xi)-2 d f=d E_{L}, \Phi_{L}(\xi)=d E_{L}+2 d f=d\left(E_{L}+2 f\right)$. The above equation (30) $L \rightarrow$ $L+2 f$. So, we can write

$$
\begin{aligned}
& \text { dif5 : }-\frac{\partial}{\partial t}\left(e^{2 f} \frac{\partial(L+2 f)}{\partial_{x_{2}}}\right)+\frac{\partial(L+2 f)}{\partial_{x_{1}}}=0, \\
& \text { dif6 }: \frac{\partial}{\partial t}\left(e^{\left.-2 f \frac{\partial(L+2 f)}{\partial_{x_{1}}}\right)+\frac{\partial(L+2 f)}{\partial_{x_{2}}}=0,}\right. \\
& \text { dif7 }: c \frac{\partial}{\partial t}\left(e^{\left.-2 f \frac{\partial(L+2 f)}{\partial_{x_{1}}}\right)}-\frac{1}{2}(a-b) \frac{\partial}{\partial t}\left(e^{\left.2 f \frac{\partial(L+2 f)}{\partial_{x_{2}}}\right)}-\frac{\partial}{\partial t}\left(e^{2 f \frac{\partial(L+2 f)}{\partial_{x_{4}}}}\right)+\frac{\partial(L+2 f)}{\partial_{x_{3}}}=0,\right.\right. \\
& \text { dif } 8:-\frac{1}{2}(a-b) \frac{\partial}{\partial t}\left(e^{-2 f} \frac{\partial(L+2 f)}{\partial_{x_{1}}}\right)-c \frac{\partial}{\partial t}\left(e^{-2 f \frac{\partial(L+2 f)}{\partial_{x_{2}}}}\right)+\frac{\partial}{\partial t}\left(e^{-2 f} \frac{\partial(L+2 f)}{\partial_{x 3}}\right)+\frac{\partial(L+2 f)}{\partial_{x 4}}=0,
\end{aligned}
$$

and these differential equations (32) is named a conformal Weyl-Euler-Lagrange mechanical system $\left(M_{4}, \Phi_{L}, \xi, F\right)$ on Walker manifold on Walker-Weyl manifold $\left(M_{4}, g, D, \nabla, J, F\right)$

\section{Computer solution of equations and graph}

The location of each object in space represented by three dimensions in physical space. Three-dimensional space is a geometric three-parameter model of the physical universe in which all known matter exists. These three dimensions can be labeled by a combination of three chosen from the terms length, width, height, depth, mass, density and breadth. Any three directions can be chosen, provided that they do not all lie in the same plane. So, each vector represents the speed and direction of the movement of air at that point. These found (30) are partial differential equation. We can solve these equations systems of motion for the routes of moving objects (30) using Maple Algebra software. This solution is implicit and will be made according to the situation in Theorem 5 and the graphics will be drawn.

Example 4. $a_{i}, b_{i}$ and $c_{i}$

$$
L\left(x_{1}, x_{2}, x_{3}, x_{4}, t\right):=x_{4} *\left((t-1) * c_{2}+c_{1}\right) * \exp (-t)+\exp (t) *\left(c_{1}+c_{2} * t\right) * x_{3}+F_{3}(t)
$$

The number of dimensions of the equation (33) will be reduced to three and behind the graphics will be drawn. First, closed function at (33) will be selected as a special. After, the figure of the equation (33) has been drawn for the route of the movement of objects in the electromagnetic field. We choose at (33) for special case of $f\left(x_{1}, x_{2}, x_{3}, x_{4}, t\right)=t, F_{3}(t)=t$ and the conditions in the Theorem 8. of $a_{i}, b_{i}, c_{i}$;

$$
L\left(x_{1}, x_{2}, x_{3}, x_{4}, t\right)=x_{4} * t * \exp (-t)+\exp (t) *(1+t) * x_{3}+t
$$




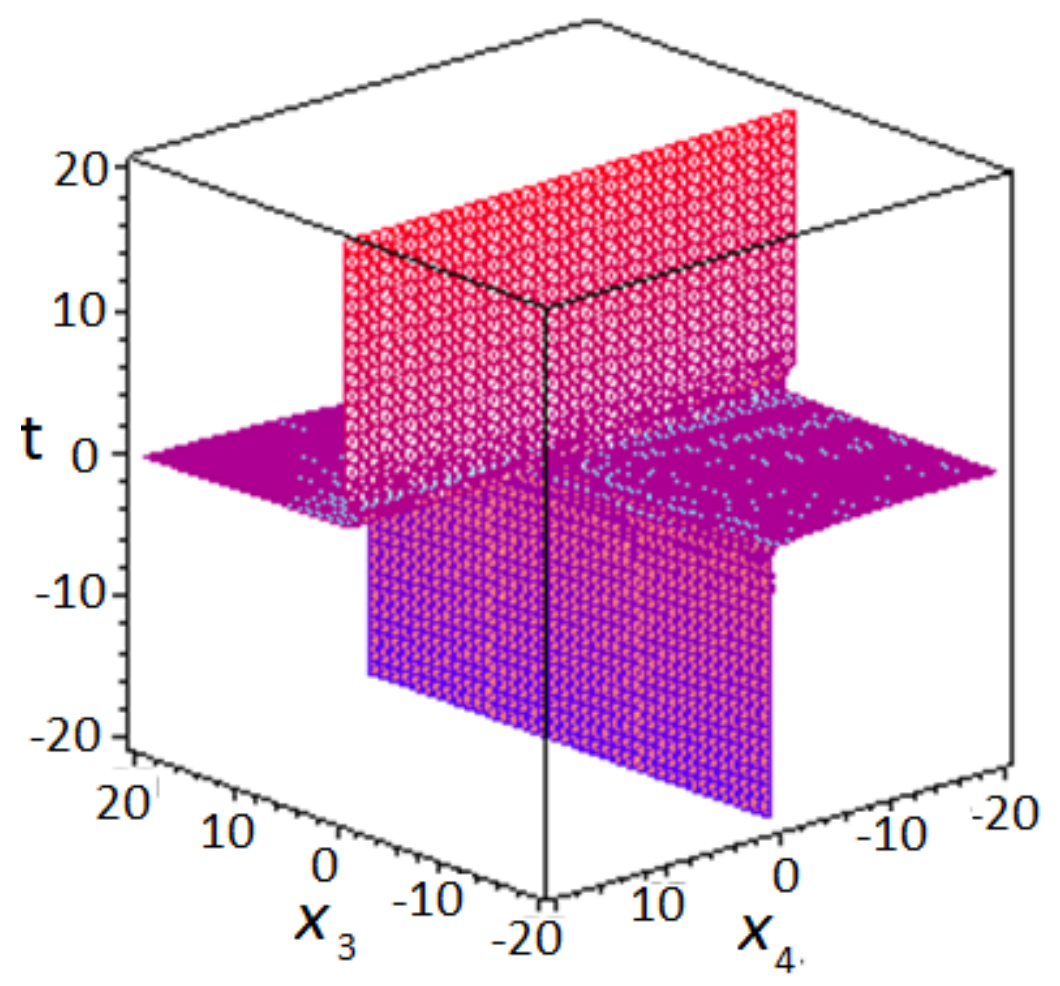

Fig. 1

\section{Discussion}

The most important advantage of this study is to obtain geodesic on 4-Walker manifolds. Thus, geodesics is to allow the calculation of linear or nonlinear distance for the orbits of moving objects. In addition, in the equations implicit solutions (33) were found using Maple Algebra software. Also, conformal Weyl-Euler-Lagrange mechanical equations (30)-(32) derived on a generalized on 4-Walker manifolds may be suggested to deal with problems in electrical, magnetically and gravitational fields force for the path of movement in above Fig.1 of defined space moving objects [29,30].

Acknowledgements. This work was supported by the agency BAP of Pamukkale University (project number: 1528). In addition, this study was presented orally at the International Conference on Anatolian Communications in Nonlinear Analysis, Abant Izzet Baysal University, Bolu, Turkey, (2013).

\section{References}

[1] J. Klein, Escapes Variationnels Et Mécanique, Ann. Inst. Fourier, Grenoble, 12, (1962).

[2] M. De Leon, P.R. Rodrigues, Methods of Differential Geometry in Analytical Mechanics, Elsevier Sc. Pub. Com. Inc., Amsterdam, (1989), 263-397.

[3] R. Abraham and J. E. Marsden, T. Ratiu, Manifolds, Tensor Analysis and Applications, Springer, (2001), 483-542.

[4] Z. Kasap and M. Tekkoyun, Mechanical Systems on Almost Para/Pseudo-Kähler-Weyl Manifolds, IJGMMP, Vol. 10, No. 5, (2013), 1-8. 
[5] Z. Kasap, Weyl-Euler-Lagrange Equations of Motion on Flat Manifold, Advances in Mathematical Physics, (2015), 1-11.

[6] A.G. Walker, Canonical Form for A Rimannian Space with A Paralel Field of Null Planes, Quart. J. Math. Oxford, Vol. 1, No. 2, (1950), 69-79.

[7] A. Salimov, M. Iscan and K. Akbulut, Notes on Para-Norden-Walker 4-Manifolds, IJGMMP, Vol. 07, No. 08, (2010), $1331-1347$.

[8] Y. Matsushita, Walker 4-Manifolds with Proper Almost Complex Structures, JGP, Vol. 55, (2005), 385-398.

[9] E. Garcia-Rio, S. Haze, N. Katayama and Y. Matsushita, Symplectic, Hermitian and Kähler Structures on Walker 4-Manifolds, J. Geom., 90, (2008), 56-65.

[10] W. Batat, G. Calvaruso and B. De Leo, On the Geometry of Four-Dimensional Walker Manifolds, Rendiconti di Matematica, Serie VII, Vol. 29, (2008), 163-173.

[11] M. Nadjafikhah and M. Jafari, Some General New Einstein Walker Manifolds, arXiv:1206.3730v1, (2012), 1-14.

[12] A.A. Salimov and M. Iscan, On Norden-Walker 4-manifolds, Note Di Matematica, Note Mat., 30, (2010), 111-128.

[13] M. Brozos-Vazquez, E. Garcia-Rio, P. Gilkey and R. Vazquez-Lorenzo, Tsankov-Videv Theory for Walker Manıfolds of Signature (2,2), SIGMA 3 (2007), 1-13.

[14] J. Davidova, J.C. Diaz-Ramosd, E. Garcia-Riob, Y. Matsushitac, O. Muskarova and R. Vazquez-Lorenzob, Almost Kähler Walker 4-Manifolds, JGP, 57, (2007), 1075-1088.

[15] M. Tekkoyun, A Survey on Geometric Dynamics of 4-Walker Manifold, JMP, 2, (2011), 1318-1323.

[16] P.R. Law, A Spinor Approach to Walker Geometry, arXiv:math/0612804v4, (2011), 1-10.

[17] Y. Matsushita, Four-Dimensional Walker Metrics and Symplectic Structures, Journal of Geometry and Physics, Vol. 52, (2004), 89-99.

[18] D. McDu and D. Salamon, J-holomorphic Curves and Quantum Cohomology, (1995).

[19] https://en.wikipedia.org/wiki/Conformal_geometry

[20] W.O. Straub, Simple Derivation of the Weyl Conformal Tensor, Pasadena, California, 2006.

[21] G.B. Folland, Weyl Manifolds, J. Differential Geometry, 4, (1970), 145-153.

[22] L. Kadosh, Topics in Weyl Geometry, Dissertationial, University of California, (1996).

[23] P. Gilkey, S. Nikčević and U. Simon, Geometric Realizations, Curvature Decompositions, and Weyl Manifolds. JGP, 61, (2011), $270-275$.

[24] http://en.wikipedia.org/wiki/Conformally_flat_manifold.

[25] P. Gilkey, S. Nikčević, Kähler-Weyl manifolds of Dimension 4, (2010), 1-11.

[26] https://en.wikipedia.org/wiki/Weyl_tensor.

[27] https://en.wikipedia.org/wiki/Weyl_transformation.

[28] M. Brozos-Vazquez, S. Nikcevic, P. Gilkey, E. Garcia-Rio and R. Vazquez-Lorenzo, The Geometry of Walker Manifolds, Vol. 2, No. 1, (2009), 1-179.

[29] H. Weyl, Space-Time-Matter, Dover Publ. (1952).

[30] B. Thidé, Electromagnetic Field Theory, http://www.physics.irfu.se/CED/Book/EMFT_Book.pdf, (2012). 